Mhicrowave Engineering

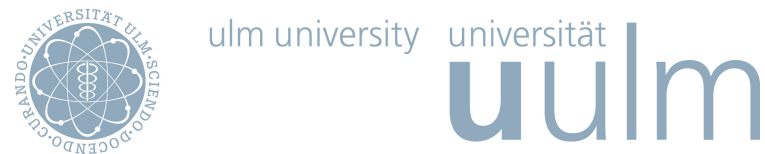

Radar Taking Off: New Capabilities for UAVs

Philipp Hügler, Fabian Roos, Markus Schartel, Martin Geiger, and Christian Waldschmidt

(c) 2018 IEEE. Personal use of this material is permitted. Permission from IEEE must be obtained for all other uses, in any current or future media, including reprinting/republishing this material for advertising or promotional purposes, creating new collective works, for resale or redistribution to servers or lists, or reuse of any copyrighted component of this work in other works.

DOI: $10.1109 / M M M .2018 .2862558$ 


\section{Radar Taking Off: New Capabilities for UAVs}

Philipp Hügler, Fabian Roos, Markus Schartel, Martin Geiger, and Christian Waldschmidt

Modern consumer and industrial unmanned aerial vehicles (UAVs) are easy to use flying sensor platforms. They offer stable flight, good maneuverability, hovering, and even waypoint flights in autopilot mode. For stabilization and localization sensors such as internal measurement units (IMUs) including gyroscope and accelerometer, barometric sensor, and global navigation satellite system (GNSS) are used. To sense the direct environment of the UAV, for instance for collision avoidance or fully automated flights, additional sensors are needed. State-of-the-art combinations of infrared sensors, ultrasonic sensors as well as vision based sensors (monocular and/or stereo vision) capture the close vicinity. Using radar sensors is advantageous, as they are able to directly sense range and velocity and are not prone to lighting conditions and contrast. With the help of a multi-channel radar, the angular information can also be extracted.

UAVs can lift a considerable payload with respect to their size. All these characteristics combined with radar sensors make them a promising tool for a large variety of applications. 


\section{Application Areas}

In industrial environments UAVs equipped with a combination of visible-light cameras and thermal cameras are used for inspection purposes for places and areas which are difficult to access. These areas include power lines, structures like bridges, and windturbines [1]. Camera sensors as well as light detection and ranging (LiDAR) sensors are used in forestry to estimate tree canopy heights [2], woody tree abundance, or even species richness [3]. Radar sensors are particularly beneficial when it comes to environments in which metallic objects need to be inspected, such as power lines or structural steel in reinforced concrete.

Parcel services are very interested in UAVs due to the possibility of fast and autonomous delivery, even in remote areas. For example, autonomous delivery flights by a Parcelcopter connecting the harbour of Norddeich and the island of Juist in the North Sea is being tested by Deutsche Post DHL Group [4] to perform emergency delivery of medication (Fig. 1). The $12 \mathrm{~km}$ flight route is used when no alternative transport such as ferries or flights are available because of low water or heavy fog. Newer approaches use a copter-based last mile delivery in alpine regions with a parcel station handling automated loading and offloading of the Parcelcopter [5]. Increasing autonomy demands reliable safety features, with radar offering excellent performance in terms of distant obstacle detection and collision avoidance, in particular under conditions when other sensors fail.

According to [6] the agriculture industry is the worldwide biggest commercial market for UAVs. UAVs can monitor field conditions and plant health with an easy to handle setup and can capture higher resolution images than satellites, even under cloudy conditions. UAVs are even used to spray fertilizer or pesticide [7] as can be seen in Fig. 2. For this kind of application the distance between the UAV and the plants must be kept constant to assure uniform spraying. This can be achieved with a radar as shown in [8]. In addition, radar is the only sensor concept which is able to perform sub-surface measu- 


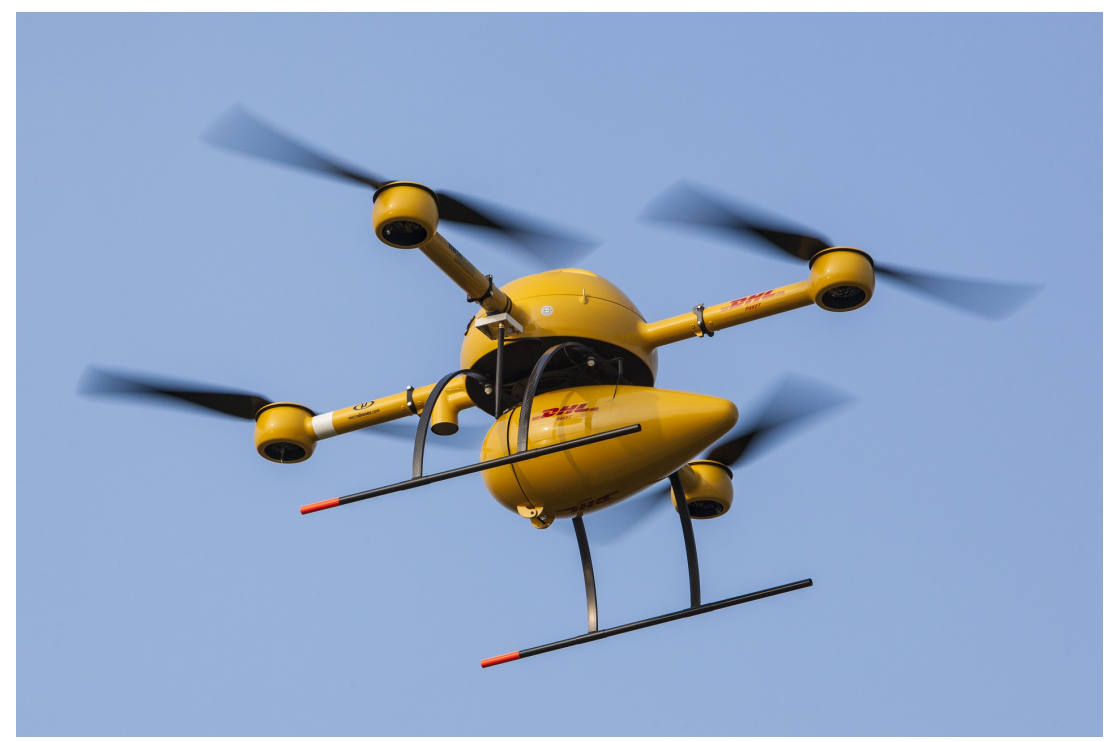

Fig. 1: DHL Parcelcopter 2.0 approaching the island of Juist, $®$ Deutsche Post AG.

rements, known as ground penetrating radar (GPR) [9]. This could be used to prevent harvesters from being damaged by buried metal objects.

Barometric sensors and GNSS can not fulfill this requirement. Visual-based sensors for altitude evaluation and collision avoidance can be operated precisely between $0.1 \mathrm{~m}$ to $8 \mathrm{~m}$ downward-looking, $0.5 \mathrm{~m}$ to $12 \mathrm{~m}$ forward-looking, and have a maximum detectable range of up to $30 \mathrm{~m}[10]$. But they often have difficulties with regular surface patterns, and the performance depends on reflectivity of the material as well as the illumination. With radar sensors one cannot only detect the altitude above ground level (AGL) but also obstacles such as vegetation [11]. Radar can directly measure the velocity of obstacles, it has a large detectable maximum range, and is very robust regarding weather influences. Because of that, drone manufacturers have started to offer radar sensors at $24 \mathrm{GHz}$ for terrain following as well as collision avoidance [7], [12]. 


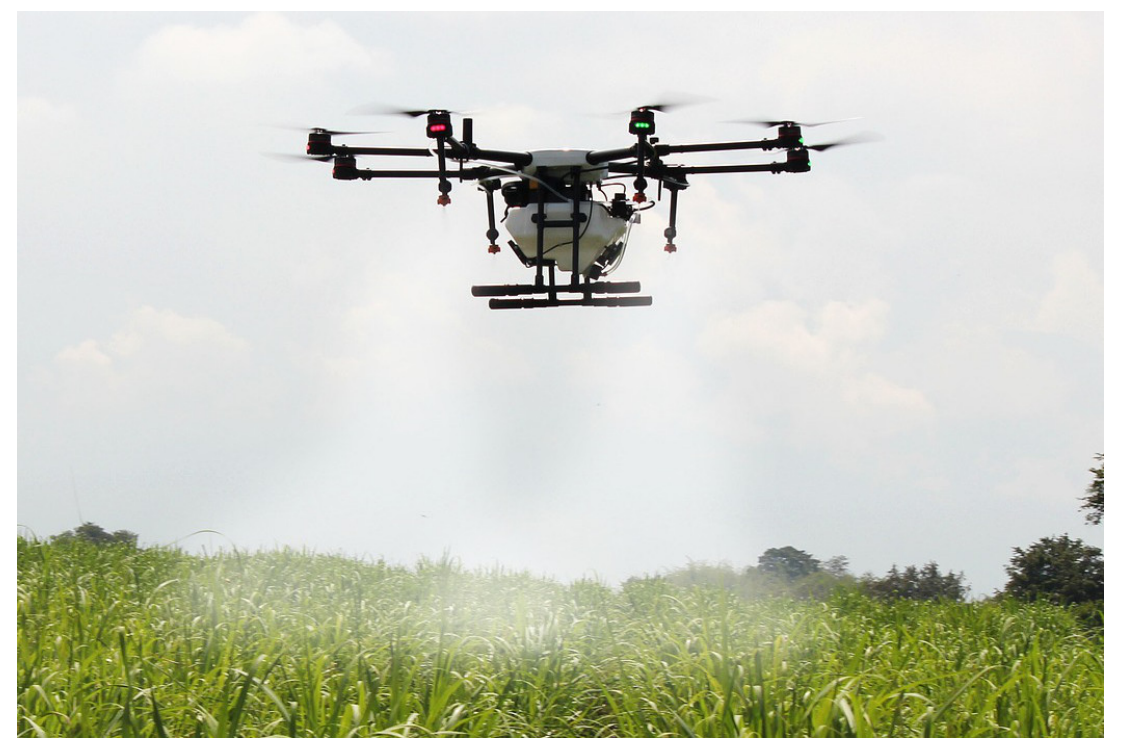

Fig. 2: Octacopter spraying a field. 


\section{UAVs and Radar: Remote Sensing and Safety Improvement}

Because of the high flexibility of UAVs they are very interesting for remote sensing applications. In the field of radar sensors, synthetic aperture radar (SAR) is used to enhance the aperture resulting in high angular resolution. Larger UAVs, e.g., with a maximum weight of $85 \mathrm{~kg}$, have already been equipped with a $94 \mathrm{GHz}$ SAR with $1 \mathrm{GHz}$ bandwidth [13]. The potential of sensing small scale features can be seen in Fig. 3, leading towards using SAR with smaller consumer drones with less than $5 \mathrm{~kg}$ weight. In [14], [15], and [16] commercial off-the-shelf pulse radar sensors of a frequency range of $3.1 \mathrm{GHz}$ to $5.3 \mathrm{GHz}$ are used for surface and subsurface imaging. A platform using a $24 \mathrm{GHz}$ frequency modulated continuous wave (FMCW) radar with $250 \mathrm{MHz}$ bandwidth for full interferometric SAR imaging and processing is evaluated in [17]. An approach for UAV-based mine detection using ground penetrating synthetic aperture radar (GPSAR) is presented in [9]. It is shown that a real-time kinematic (RTK) GNSS is needed for the demanding position accuracy to use a $1 \mathrm{GHz}$ to $4 \mathrm{GHz}$ ultra wideband FMCW radar to detect targets with low reflectivity.

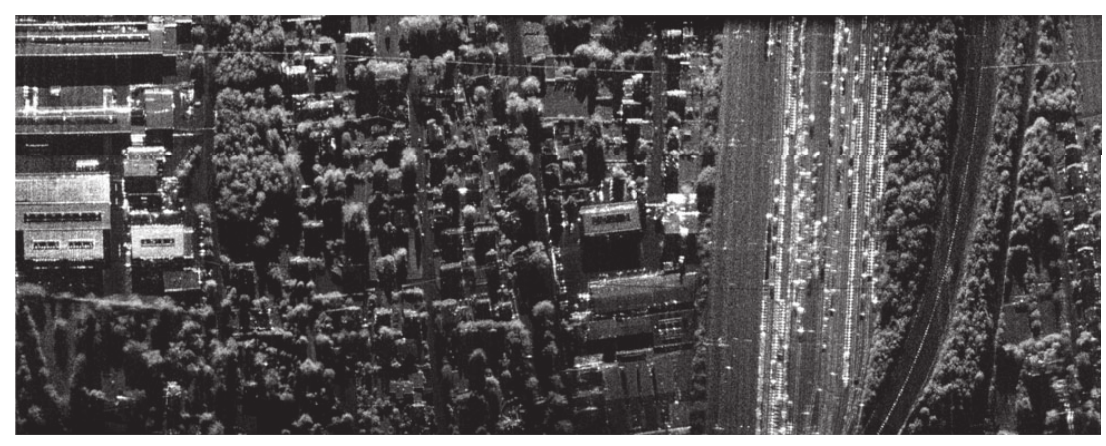

Fig. 3: UAV SAR image for urban terrain taken with MIRANDA radar, offering $1 \mathrm{GHz}$ bandwidth. Image taken from [13]. 

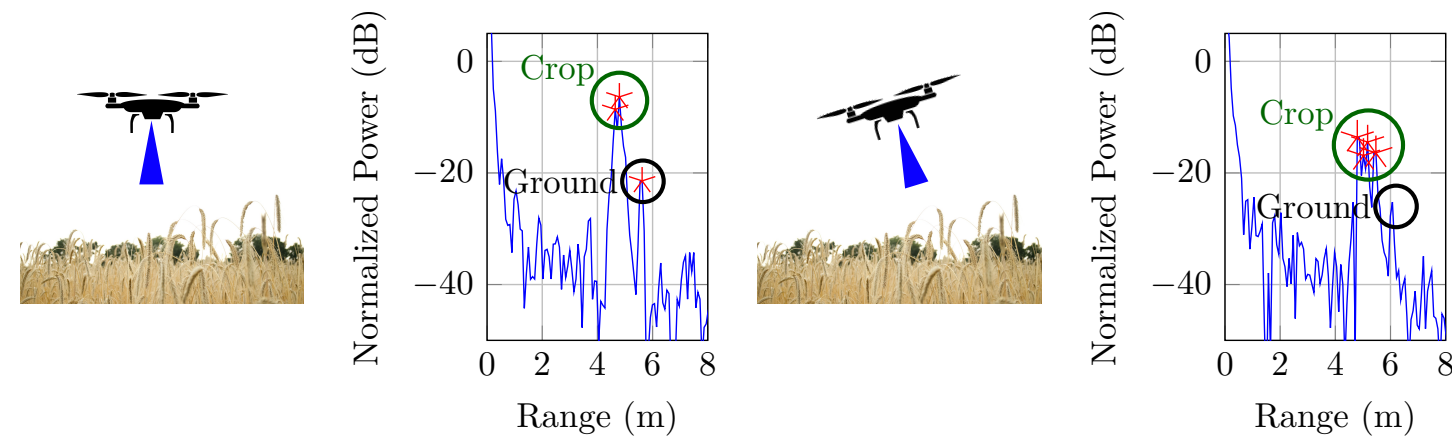

(a) Roll angle $0^{\circ}$.

(b) Roll angle $17^{\circ}$.

Fig. 4: Influence of the roll angle to altitude AGL measurements for a $77 \mathrm{GHz} F M C W$ radar altimeter, (a) with an angle of $0^{\circ}$ and (b) $17^{\circ}$. Detected targets are marked with $(*)$.

Besides remote sensing, radar can also be used to improve safety features for UAVs. Radar can detect obstacles for collision avoidance and surface monitoring even in lowcontrast situations and under severe surrounding conditions, e.g., with back light.

An easy way to detect moving targets is to use a Doppler radar as in [18], which is operating in the $\mathrm{X}$-band at a frequency of $10.5 \mathrm{GHz}$. In addition to the detection of moving targets, Doppler signatures are used to identify previously characterized aircraft. However, this setup is not suitable to evaluate the distance to the targets. With FMCW modulation, the distance as well as the velocity can be measured. Measurements in [19] show the potential of a $24 \mathrm{GHz}$ FMCW radar with $1 \mathrm{GHz}$ bandwidth for potential intruder and ground target detection. But the device in this case was not mounted on a UAV but on a stationary and moving ground station.

Sensing the altitude AGL with a $24 \mathrm{GHz}$ FMCW radar and fusing it with accelerometer data is validated indoors using a motion capture system consisting of 24 cameras [20]. An in-depths analysis of a $77 \mathrm{GHz}$ radar-based altimeter is given in [11]. With $2 \mathrm{GHz}$ bandwidth, 15 measurements per second, and an effective isotropic radiated power (EIRP) of approximately $1 \mathrm{~W}$, a number of different experiments have been performed. It is shown, that a constant false alarm rate (CFAR) algorithm can reliably detect altitudes 


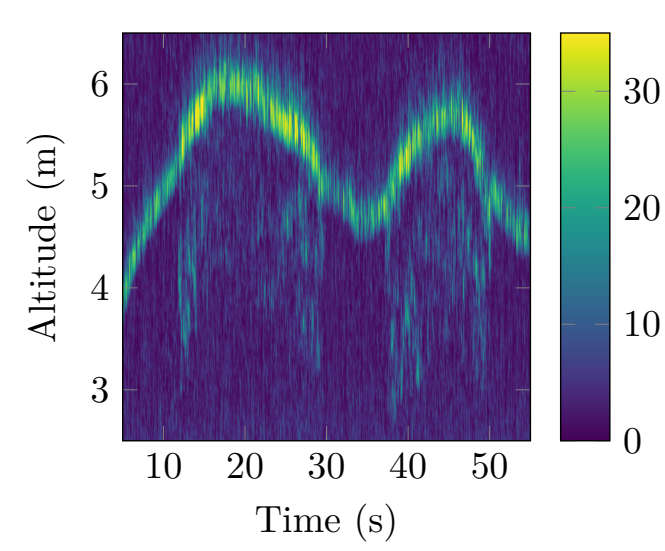

(a) Radar data from flight above cornfield.

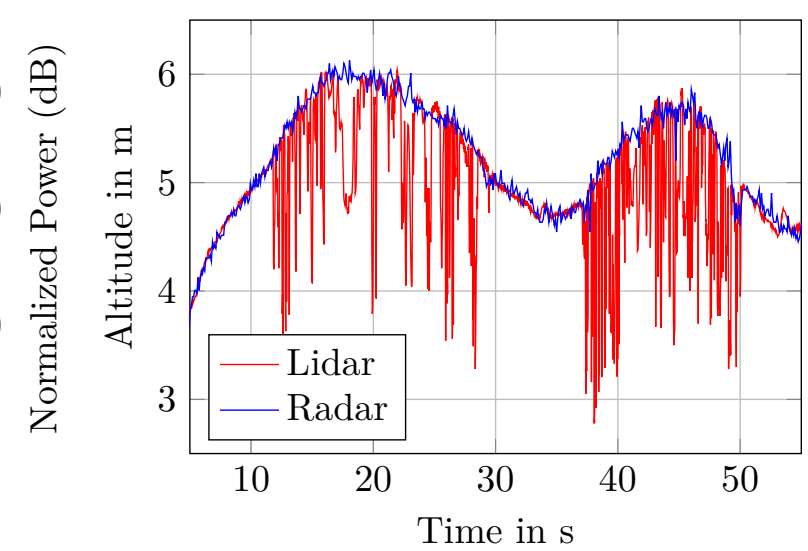

(b) Altitude AGL from radar data with particle filter versus LiDAR data.

Fig. 5: Comparison of altitude AGL measurements of a $26 \mathrm{GHz}$ pulse correlation radar with particle filter and LiDAR when flying over a cornfield [8].

up to $40 \mathrm{~m}$ over grass. In addition, the influence of tilting caused by acceleration of the UAV is examined, an example for a roll angle of $17^{\circ}$ is depicted in Fig. 4. In spite of the degradation of the signal-to-noise ratio (SNR) by $10 \mathrm{~dB}$, not only the ground but also the crop is visible. The same is valid for other vegetation such as trees. Due to the high bandwidth, even single steps of stone masonry (between $20 \mathrm{~cm}$ to $25 \mathrm{~cm}$ high) can be resolved.

In order to reduce hardware complexity and therefore achieve lower costs, a pulse correlation radar can be used for altitude AGL estimation. In [8] a $26 \mathrm{GHz}$ radar in conjunction with a particle filter to track the altitude AGL with an update rate of up to $40 \mathrm{~Hz}$ is compared with a low cost LiDAR and an RTK GNSS. As can be seen in Fig. 5(b), in the presence of vegetation between the UAV and the ground, the radar outperforms the LiDAR, which measures the distance to vegetation instead of the altitude AGL.

To improve obstacle detection and collision avoidance, measuring radial velocity and the distance of targets is not enough. It would be useful to have at least the azimuth angular or direction-of-arrival (DoA) information to obtain two-dimensional spatial in- 
formation of the location of the potential obstacle. The increasing use of radar in the automotive industry $[21,22]$ has lead to a considerable decrease in cost and a high level of integration. Complete radar frontends, including antennas, in a single package are available in the industrial, scientific, and medical (ISM)-bands at $60 \mathrm{GHz}$ and $122 \mathrm{GHz}$ for industrial applications [23-25], making the realization of small form factor and lightweight radar sensors feasible for UAV applications. 


\section{Seeing Even More with Imaging Radar}

For collision avoidance, an imaging radar has to fulfill several requirements. The separability of targets in range direction is important, therefore, a range resolution in the single-digit centimeter range is desirable. Assuming a maximum velocity $v_{\text {drone }}$ of the $\mathrm{UAV}$ of $\pm 15 \frac{\mathrm{m}}{\mathrm{s}}$ an unambiguous velocity of at least $v_{\text {drone }}$ for stationary and $2 v_{\text {drone }}$ for moving targets should be achieved. For classification purposes, a high Doppler resolution would be advantageous. In order to gain high angular resolution an adequate number of channels is needed.

\subsection{Extracting Range and Velocity: Used Modulation Format}

Because of the expected multi-target scenario, an FMCW radar with fast ramps, also called chirp-sequence frequency modulation [26], is chosen as the operating principle of the imaging radar. Transmitting several saw-tooth-shaped ramps (called a block), range and velocity can be extracted using a 2D-Fourier transform (FT) [27]. Because of the fast ramps, the velocity influence can be neglected for the range calculation, realized by a first FT over each ramp. With a second FT over several ramps, the motion induced phase change from ramp to ramp can be extracted, which is proportional to the velocity. At a carrier frequency $f_{\mathrm{c}}$ of $76.5 \mathrm{GHz}$ a bandwidth $B$ of $2 \mathrm{GHz}$ is chosen to achieve a range resolution $\Delta r$ of $75 \mathrm{~mm}$. The maximum unambiguous velocity $v_{\max }$ is limited by the ramp repetition interval $T_{\mathrm{RRI}}$ and is set to $60 \mu \mathrm{s}$, resulting in $\pm 16 \frac{\mathrm{m}}{\mathrm{s}}=v_{\max }$

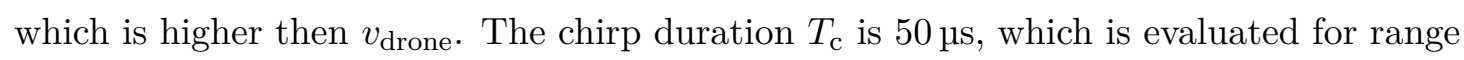
extraction. The overall measurement time (time of a single block) determines the velocity resolution $\Delta v$ and results form the number of chirps $L$ per block multiplied with $T_{\mathrm{RRI}}$. A summary of the used modulation parameters and the resulting radar performance is

listed in Tab. 1. In the following experiments, only stationary targets or slow moving targets are considered, thus, the used $T_{\mathrm{RRI}}$ is sufficient. For application with higher relative velocity, decreasing $T_{\mathrm{RRI}}$ and $B$ by a factor of two would double $v_{\max }$ and half 


\begin{tabular}{ll} 
Parameter & Value \\
\hline Carrier frequency $f_{\mathrm{c}}$ & $76.5 \mathrm{GHz}$ \\
Bandwidth $B$ & $2 \mathrm{GHz}$ \\
Chirp duration $T_{\mathrm{c}}$ & $50 \mu \mathrm{s}$ \\
Ramp repetition time $T_{\mathrm{RRI}}$ & $60 \mathrm{\mu s}$ \\
Number of chirps $L$ & 258 \\
& \\
Radar performance & $75 \mathrm{~mm}$ \\
\hline Range resolution $\Delta r$ & $\pm 16 \frac{\mathrm{m}}{\mathrm{s}}$ \\
Max. unambiguous velocity $v_{\max }$ & $\pm 13 \frac{\mathrm{cm}}{\mathrm{s}}$
\end{tabular}

Tab. 1: Summary of the used modulation parameters and the resulting radar performance in range and velocity.

$\Delta r$. This would not change the maximum expected intermediate frequency (IF), and therefore, the sampling frequency could remain the same.

\subsection{The Way to Imaging Radar: DoA Estimation}

For high angular resolution a large aperture is needed. Because of the limited space and weight when attached to a UAV a multiple-input multiple-output (MIMO) radar can be used to generate a large virtual aperture with a limited number of transmitters and receivers. Several orthogonal waveforms for FMCW radars are discussed in [28]. Here time-division multiplexing (TDM) is applied, achieving orthogonality by switching transmitters consecutively on and off, with only one transmitter is active at the same time. For the actual DoA estimation there are several techniques available, as discussed in [29], [30], and [31].

The radar has four transmitters and eight receivers on one printed circuit board (PCB). The antenna system is placed on an extra PCB to allow different antenna setups without the need of re-manufacturing the radar board. The transition between both PCBs is realized by a PCB-to-waveguide transition using a coplanar patch radiating in the $\mathrm{H}$ plane of an WR10 waveguide, cf. [32]. The transition is available in two versions, one for down- and one for forward-facing antenna arrays. 


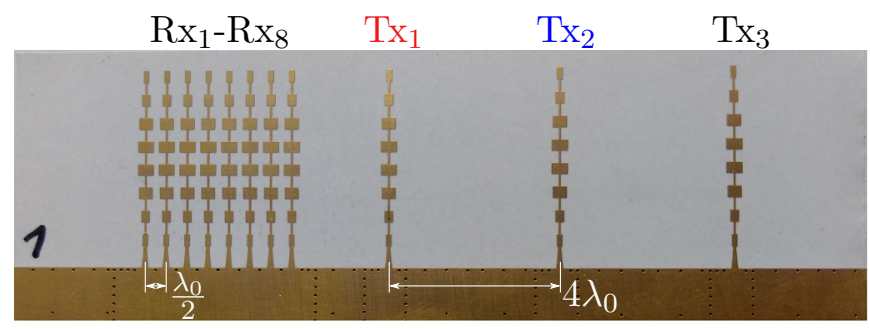

(a) Photograph of the fabricated antenna array on PCB.

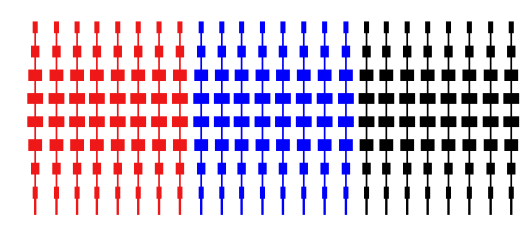

(b) Resulting virtual aperture.

Fig. 6: (a) Fabricated and (b) resulting virtual aperture of the antenna array with three transmitters $(\mathrm{Tx})$ and eight receivers $(\mathrm{Rx}) . \lambda_{0}$ is the free-space wavelength.

In Fig. 6(a) the antenna frontend used for the following experiments is depicted. It uses three transmitters and eight receivers. The position of the elements are chosen in such a way, that by the convolution of the transmitter positions with the receiver positions, a 24 element uniform linear array (ULA) is obtained as a virtual aperture (Fig. 6(b)) [33]. The ULA has a $\pm 90^{\circ}$ ambiguity-free region, low side-lobe levels, and straight-forward DoA estimation. The single channels are realized with series-fed patch antennas with $12 \mathrm{dBi}$ gain and a $3 \mathrm{~dB}$ beamwidth of $\pm 40^{\circ}$ in azimuth and $\pm 7^{\circ}$ in elevation, which is considered as the minimum field of view (FoV).

\subsection{TDM MIMO Limitations and their Compensation}

TDM MIMO comes with low hardware efforts compared to other orthogonal waveforms, but introduces a phase error when a movement, either by the targets and/or the radar, is present during switching between transmitters. This phase error leads to inaccurate or even completely unusable angular estimation. One way to overcome this problem is to realize a virtual aperture with overlapping elements where the phase offset between the different active transmitters can directly be measured [34]. As can be seen in Fig. 6(b) no overlapping element is used here to maximize the virtual aperture. The phase error in this case is fully compensated at no extra effect by adapting the FT processing as presented in [35].

For UAV-based collision avoidance, the TDM scheme reduces the maximum unambi- 
guous velocity

$$
v_{\max }= \pm \frac{c}{2 f_{\mathrm{c}}} \frac{1}{2 M T_{\mathrm{RRI}}}
$$

by the number of used transmitters $M$. With the solution presented in [36] the same unambiguous Doppler velocity as for the single-input multiple-output (SIMO) case can be achieved with phase evaluation of the virtual aperture.

Applying these compensation techniques to the signal processing chain of the TDM MIMO radar, all previously specified requirements for UAV-based collision avoidance and environment sensing are fulfilled.

\subsection{Measurement System and Target Extraction}

The UAV used for the measurements is a DJI Matrice 600 Pro hexacopter. It offers high reliability with three redundant IMUs and GNSSs systems and can lift a payload of up to $6 \mathrm{~kg}$ with a flight time of approximately $15 \mathrm{~min}$. If higher positional accuracy is needed, an RTK GNSS can be mounted. The telemetry data provided by the drone is captured at $50 \mathrm{~Hz}$. The imaging radar as well as a custom-made measurement PC are mounted to the drone. The raw time-domain data is simultaneously sampled with an eight channel analog-to-digital (AD) converter card connected to a peripheral component interconnect express $(\mathrm{PCI}) 3.0 \times 8$ slot. The sampling clock is coherently derived from the radar reference clock. For each transmitted set of frequency chirps an industrial camera mounted to the UAV is hardware triggered to obtain a picture of the measured scenario. The PC is build around a micro-ITX mainboard and features a low thermal design power (TDP) Core i7 CPU with 16 GB RAM and an SSD (connected to PCIe $3.0 \times 4$ ) for fast data rates of up to $1.5 \mathrm{~GB} / \mathrm{s}$. The $\mathrm{PC}$ with the imaging radar mounted on top is depicted in Fig. 7.

Over the video link of the Matrice 600 Pro live status information of the sampling card, telemetry script, and the overall measurement status as well as the actual camera image are transmitted to a smartphone connected to the UAV remote controller. The 


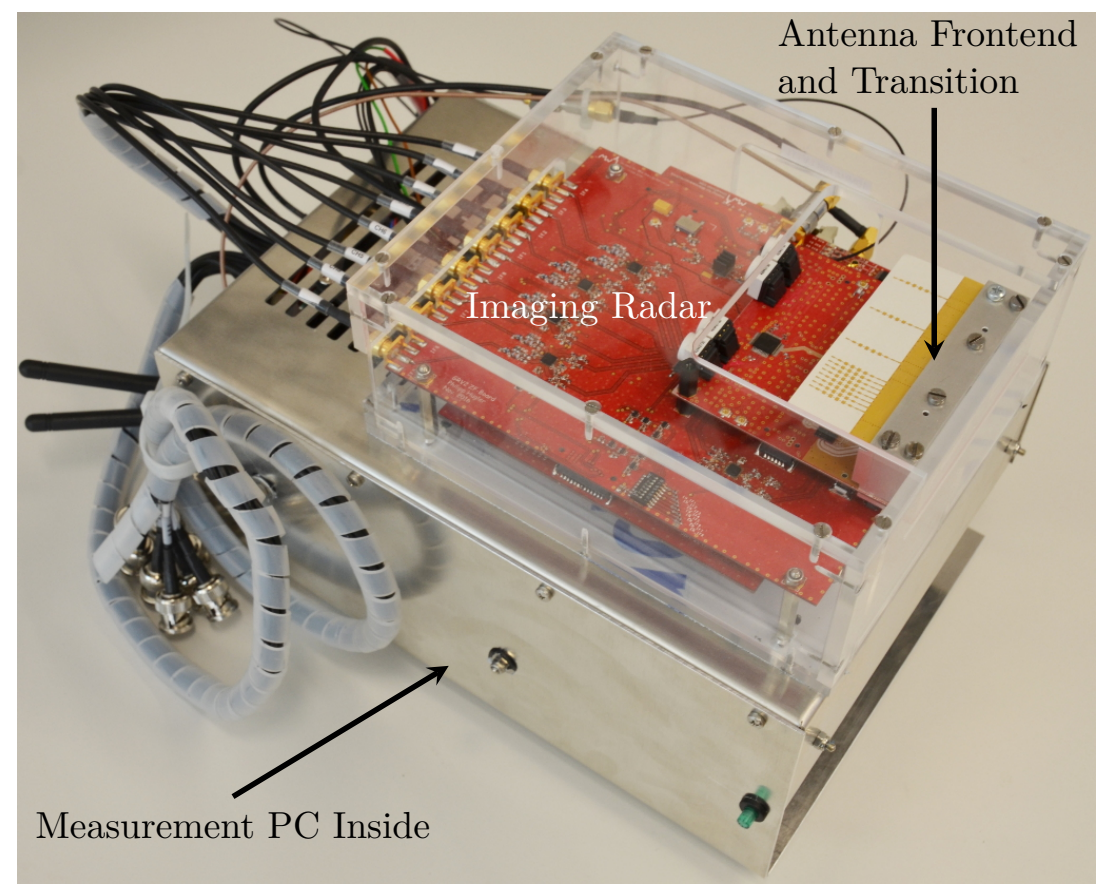

Fig. 7: Picture of the imaging radar mounted to the measurement PC.

complete system and additional sketches of the UAV showing the covered area by the FoV of the antennas are depicted in Fig. 8.

After AD conversion of the raw data, the time-domain data is zero-padded by a factor of two, and the data of a complete block, consisting of 258 frequency chirps, is arranged in a $3 \mathrm{D}$ array. A Hann window is applied to all three dimensions. A linear prediction algorithm is used and the DoA estimation is applied to each range-Doppler cell for better visualization using a Bartlett beamformer. With a 3D-FT the range, velocity, and angular data can be calculated. A CFAR algorithm is applied to the range-velocity data to extract possible targets. Radar measurement, video image, and the telemetry dataset are synchronized by a GNSS time stamp. The radar specifications are summarized in Tab. 2.

In the following section, two scenarios with the imaging radar facing down are presented. After that, two different scenarios for obstacle avoidance with the imaging radar facing forward are investigated. 


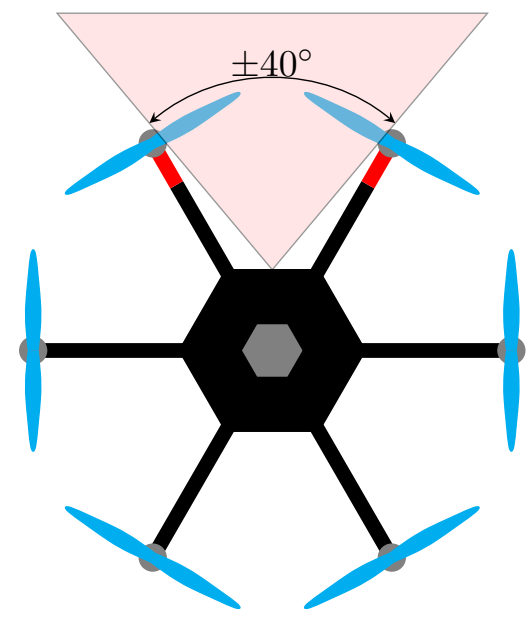

(a)

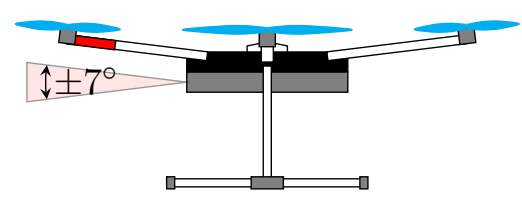

(b)

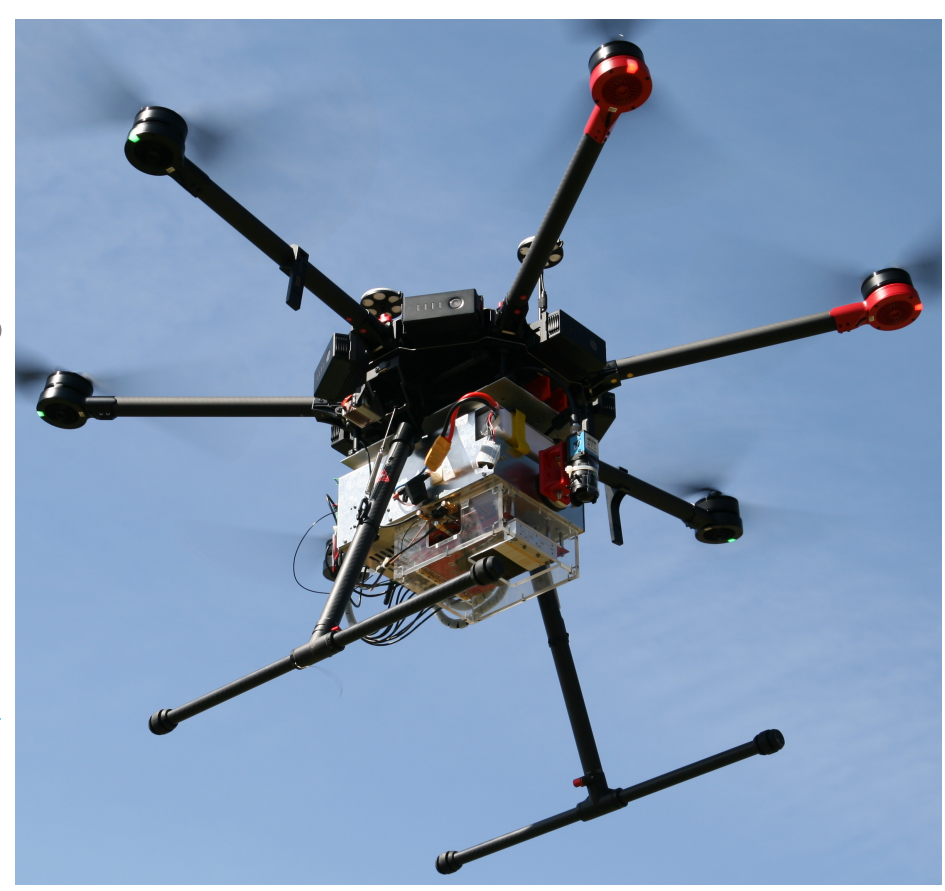

(c)

Fig. 8: The minimum FoV of the imaging radar is shown in (a) top view (azimuth) and (b) side view (elevation). The complete UAV with the mounted imaging radar sensor and the measurement PC is depicted in (c).

\begin{tabular}{ll} 
Parameter & Value \\
\hline Modulation & Chirp-sequence \\
Sampling frequency & $20 \mathrm{MHz}$ \\
ADC resolution & $14 \mathrm{bit}$ \\
Update rate & $15 \mathrm{~Hz}$ \\
MIMO scheme & TDM \\
Number of transmitters & $3 \mathrm{used}, 4$ available \\
Number of receivers & 8 \\
EIRP & $17 \mathrm{dBm}$ \\
\hline Virtual aperture & 24 element ULA \\
Antenna gain & $12 \mathrm{dBi}$ \\
min. FoV azimuth & $\pm 40^{\circ}$ \\
min. FoV elevation & $\pm 7^{\circ}$
\end{tabular}

Tab. 2: Summary of the radar parameters including information about the MIMO scheme used, available number of Tx and Rx, and information on the antenna. 


\subsection{Downward-Facing Measurements}

In the case of downward-facing measurements the antenna beam in azimuth covers the $x$ and the antenna beam in elevation covers the $y$ axis (compare Fig. 9). This setup can be used to obtain terrain profiles, sense present vegetation, find suitable landing places for planned or emergency landings, and to keep a constant altitude AGL.

The first scenario presents a UAV take-off on a meadow. The ground-profile $(x-z-$ plane) is shown in Fig. 10(a). With the measured roll-angle of approx. $0.5^{\circ}$ from IMU data, it can be seen that the UAV take-off area is located on a slightly descending surface. Additional targets at a distance of up to $1 \mathrm{~m}$ are also visible. These arise from direct transmitter-receiver-leakage and from the landing skid which is not yet retracted, cf. Fig. 10(c).

From the range-velocity plot in Fig. 10(b) the take-off movement is clearly observable. Several targets with velocities around $0.5 \frac{\mathrm{m}}{\mathrm{s}}$ are visible in the range from $-1.8 \mathrm{~m}$ to $-3 \mathrm{~m}$. In contrast to the DoA estimation, only the radial distance and velocity can be evaluated, hence this distribution of targets is expected. Targets which are further away also have a lower velocity. This is caused by the Doppler dependency on the cosine of the incidence angle. In addition, the landing skid being retracted is visible in the range-velocity diagram, and is marked red in Fig. 10(b). The landing skid is very close, thus multiple reflections between it and the radar are present. This could be used to check if the landing skid is ready before touchdown.

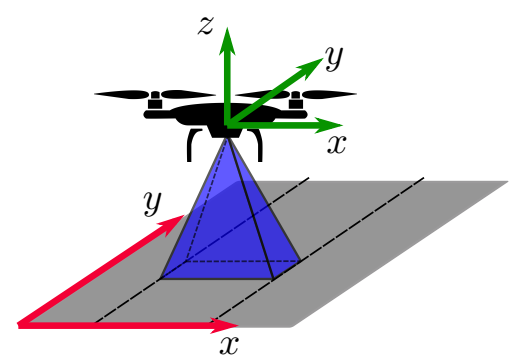

Fig. 9: Used coordinate system for all measurements with the illuminated area sketched in the case of a downward-facing antenna array. 


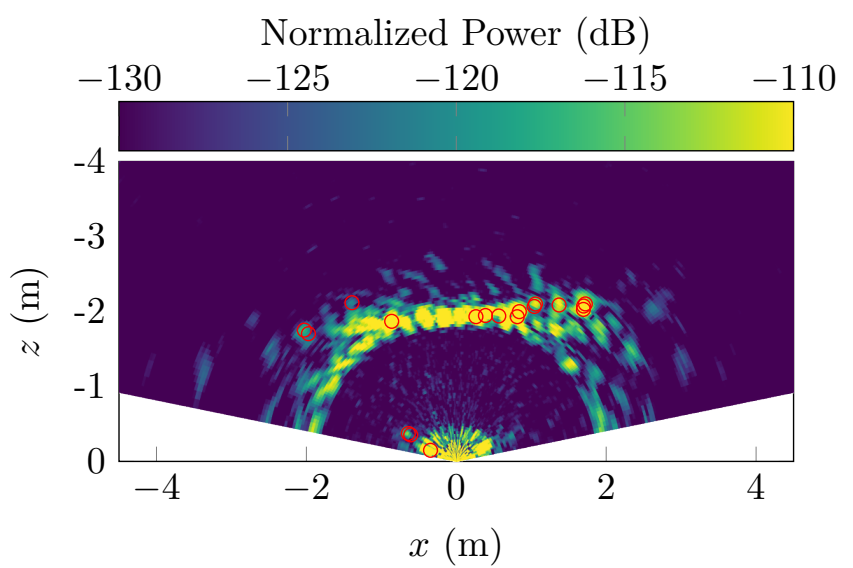

(a) Ground profile in the $x$-z-plane.

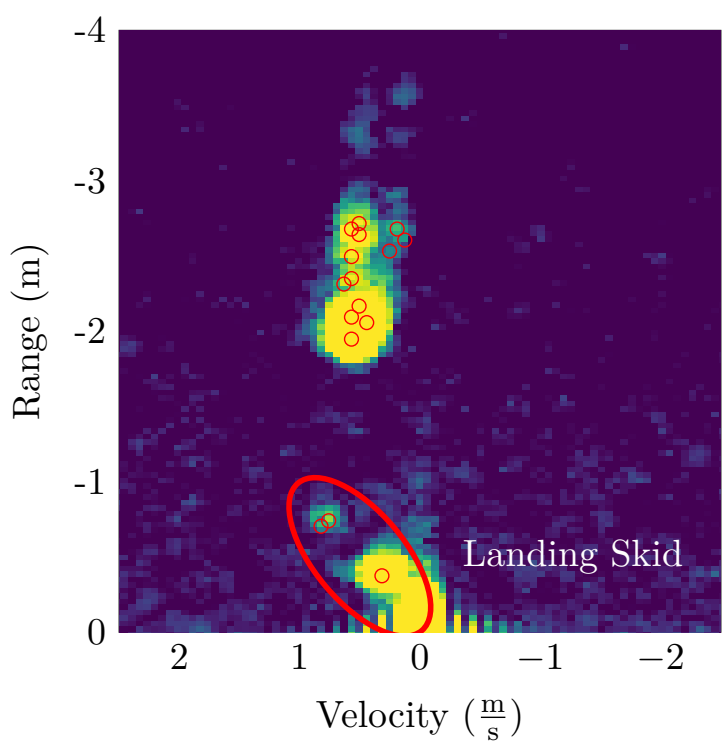

(b) Range-velocity diagram.

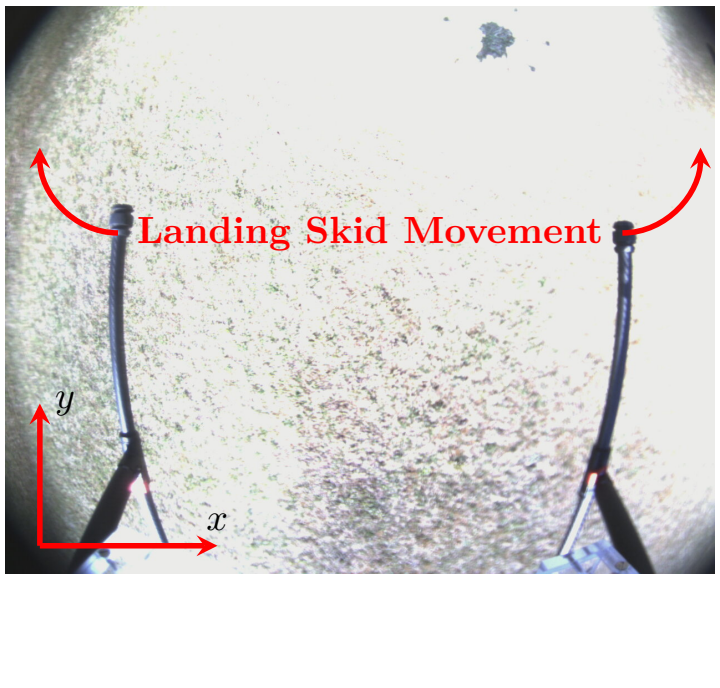

(c) Bird eye view.

Fig. 10: Measurement of a take-off with the landing skid being retracted, depicted as (a) a ground profile plot, (b) in range-velocity, and (c) a picture of the scenario. Detected targets are marked with (०). 
A measurement with the UAV flying over a $1.2 \mathrm{~m}$ high stepped stone masonry is considered next. The UAV is moving with a constant velocity in the $y$-direction. The ground profile has two clearly distinguishable levels and there are nearly no velocity components present. The measured ground profile is depicted in Fig. 11(a) and the scenario from side view in Fig. 11(b). Two distinct levels at approximately $-4.2 \mathrm{~m}$ and $-5.4 \mathrm{~m}$ are present as expected. In the encircled area, the steep masonry can be seen. Compared to the previous measurement in Fig. 10(a), it is obvious that the landing skid is retracted and not visible as a close target anymore. Considering the range-velocity diagram in Fig. 11(a), wide spread targets in range direction with almost no velocity components are visible between $-4 \mathrm{~m}$ and $-6 \mathrm{~m}$.

This experiment shows that an imaging radar can provide very detailed ground profiles with a single measurement. Since UAVs are trending towards more autonomy, UAVs also have to perform autonomous landing maneuvers in case of emergency. A UAV equipped with an imaging radar could determine a suitable landing area based on the measured ground profile. 


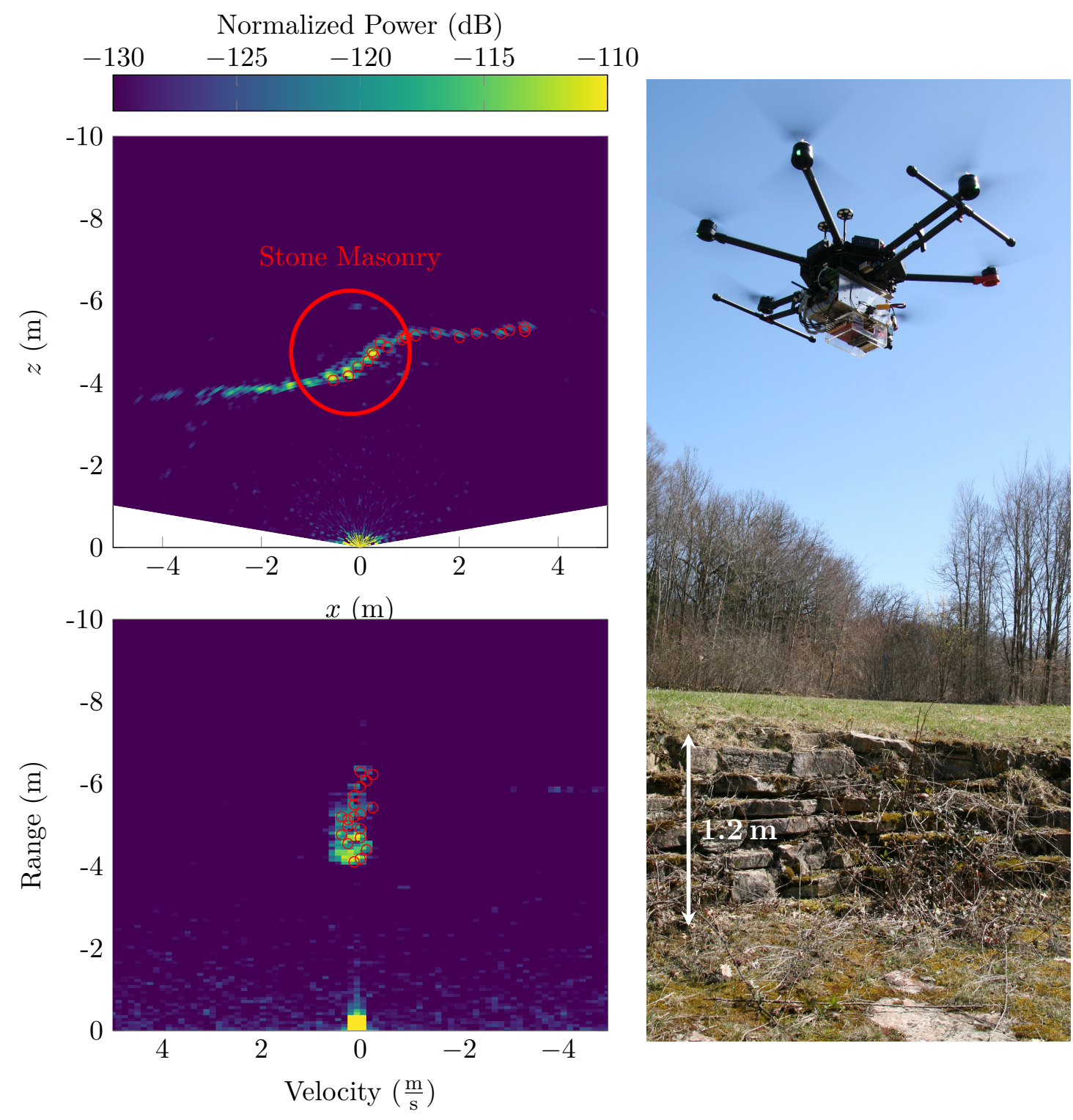

(a) Radar measurements in the $x$-z-plane and the range-velocity diagram.

(b) Picture of the UAV flying alongside the stone masonry.

Fig. 11: Measurement of the ground profile of a stone masonry, depicted (a) as a ground profile in the $x$ - $z$-plane and a range-velocity plot, and (b) a picture of the scenario. Detected targets are marked with $(\circ)$. 


\subsection{Forward-Facing Measurements}

With the imaging radar in the forward-facing orientation, scenarios for obstacle detection as well as collision avoidance are considered. In this setup, the antenna beam in azimuth covers again the $x$-axis, and the antenna beam in elevation the $z$-axis, as depicted in Fig. $8(\mathrm{a})$ and $8(\mathrm{~b})$. The orientation of the camera is adjusted in order to have a first person view (FPV) of the UAV and the radar.

At first, a measurement with the UAV moving alongside a high metal fence with a speed of approximately $5 \frac{\mathrm{m}}{\mathrm{s}}$ is considered (Fig. 12). A picture from the FPV perspective is given in Fig. 12(c). Because of the FoV of $\pm 40^{\circ}$ in the $x$-direction, it is expected that even close fence posts should be visible in the $x$-y-plane. The subsequent fence posts and other parts of the fence between the posts are visible for distances over $10 \mathrm{~m}$. From the range-velocity diagram in Fig. 12(b), the targets are distributed over the velocity in a cosine-shaped manner, which can be derived from the dependency of the Doppler frequency on the cosine of the angle of arrival. 


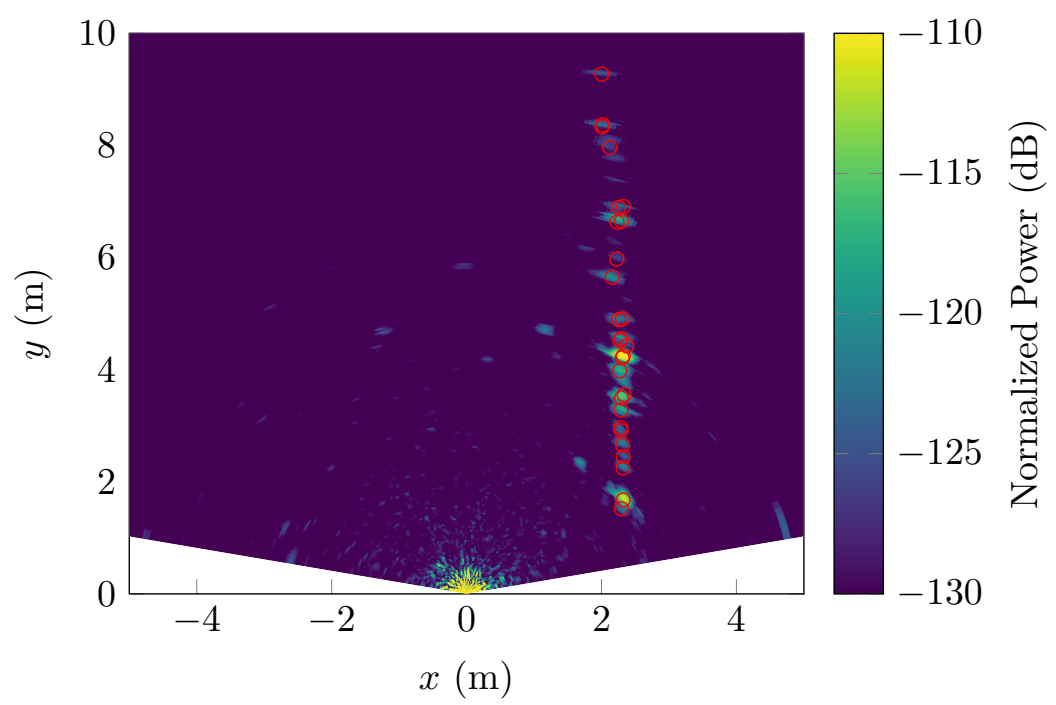

(a) Front view in $x$-y-plane.

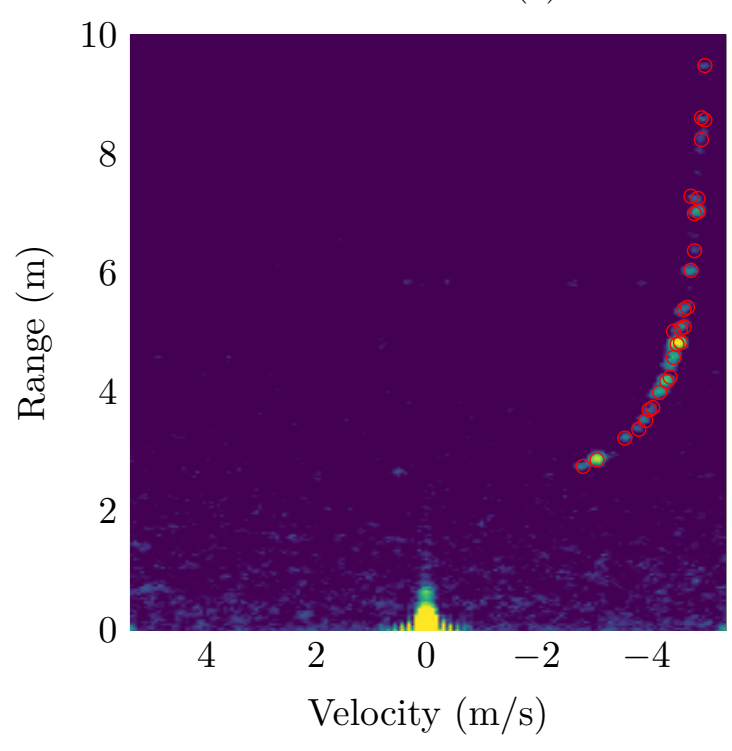

(b) Range-velocity diagram.

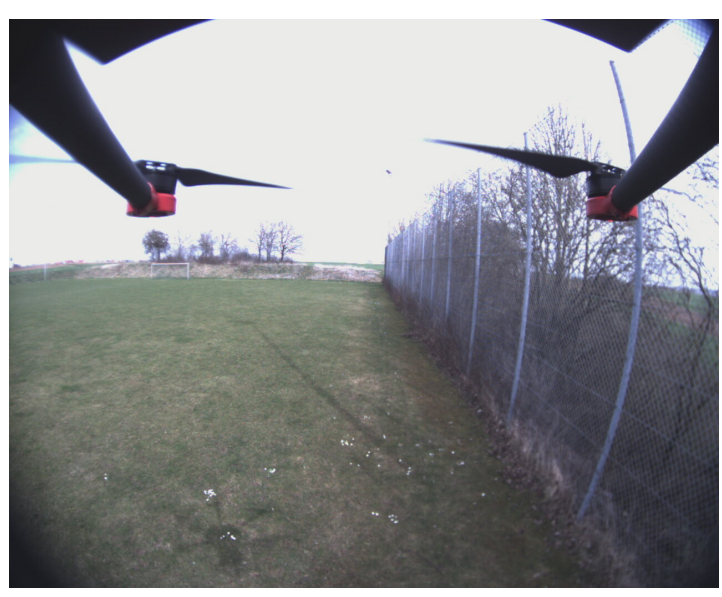

(c) Photo from first person view.

Fig. 12: UAV with forward-facing imaging radar moving alongside a high metal fence. Measurements depicted in (a) for the $x$-y-plane, in (b) for range-velocity, and in (c) a picture from first person view. Detected targets are marked with (०). 
Second, collision avoidance capability is investigated with a moving target, while the UAV with the mounted imaging radar is hovering at a fixed altitude. A smaller second UAV, a DJI Phantom 3, with a body diameter of $30 \mathrm{~cm}$ excluding propellers is used as a target. The target is flying by from rear right, heading to front left. Two snapshots separated by $740 \mathrm{~ms}$ from FPV perspective are depicted in Fig. 13(a), with the lefthanded column being the earlier point in time. As can be observed in the $x$ - $y$-diagram in Fig. 13(b), even this relatively small UAV can clearly be detected and located. The radial velocity can be extracted from the range-velocity diagram in Fig. 13(c). From snapshot to snapshot, the route of the target can be followed. Several consecutive measurements could be evaluated to estimate the targets orientation and trajectory. With the trajectory and the direct velocity measurement, the flight controller can monitor if a collision is imminent and perform an evasive maneuver. 

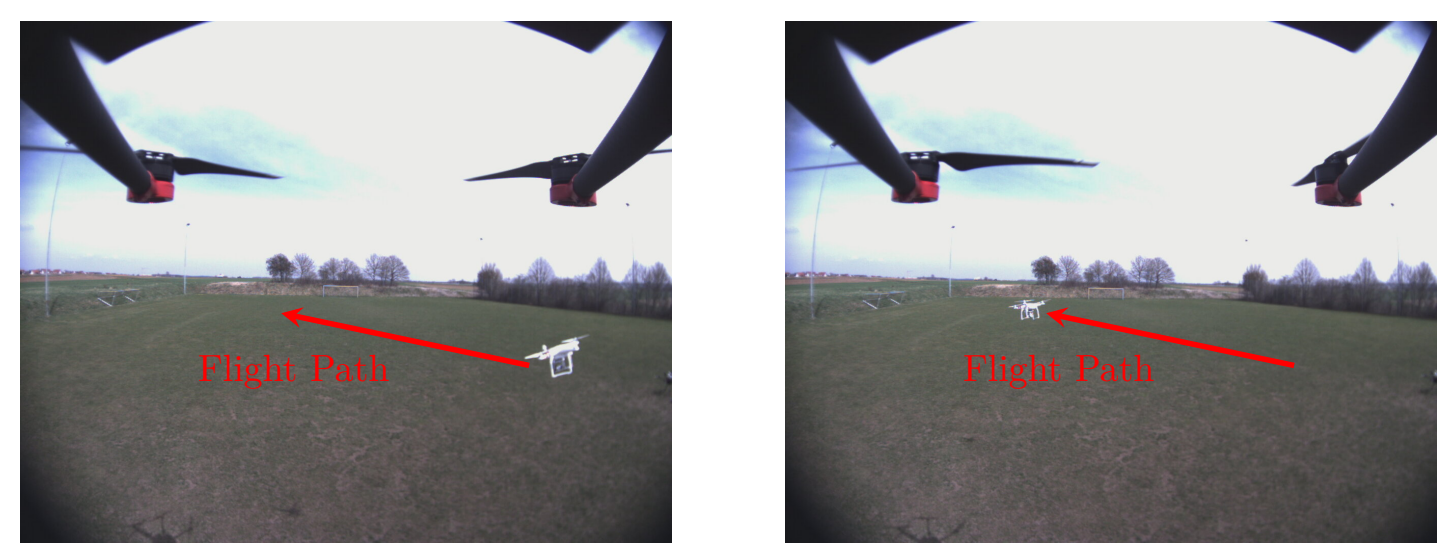

(a) Camera image from first person view.

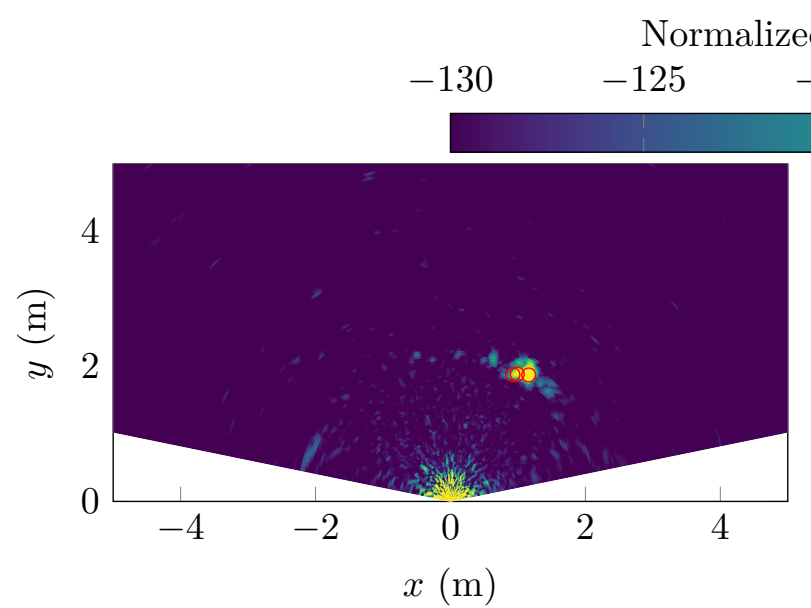
Power $(\mathrm{dB})$

(b) Radar measurements in $x$ - $y$-plane.
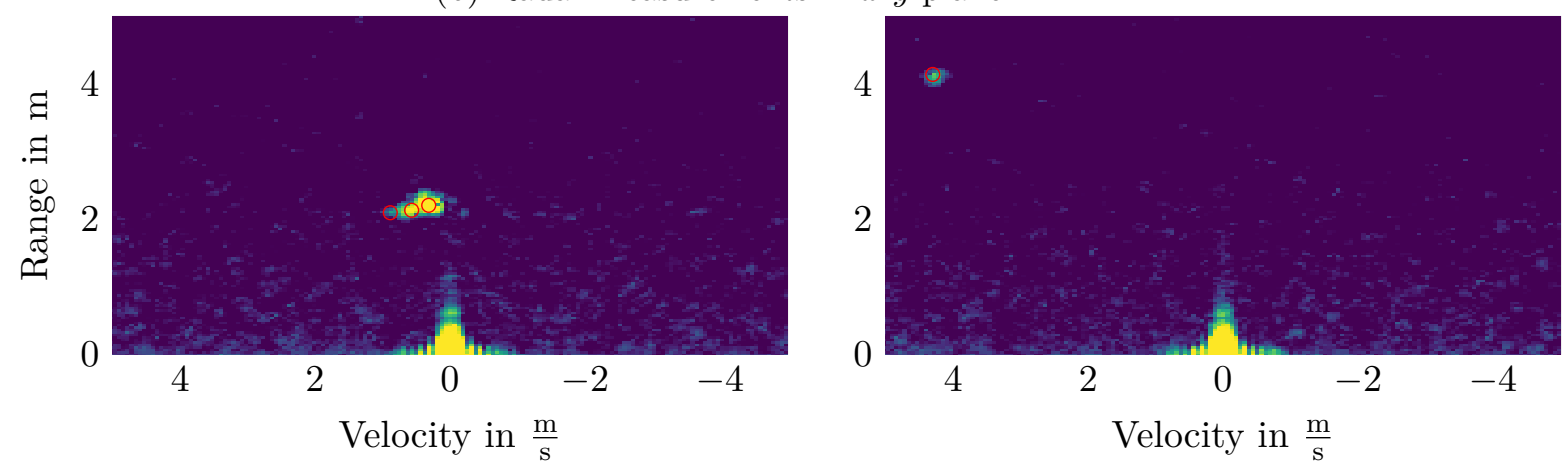

(c) Radar measurements in range and velocity.

Fig. 13: Small drone (DJI Phantom 3) with a diameter of approximately $30 \mathrm{~cm}$ excluding propellers passing by from right to left. Two snapshots separated by $740 \mathrm{~ms}$ are depicted from FPV in (a), radar measurements in $x$-y-plane in (b), and (c) range-velocity diagram. Detected targets are marked with (०). 


\section{Conclusion}

High flexibility and easy usability boost the use of UAVs in consumer and industrial applications, ranging from autonomous parcel delivery to agriculture. Utilizing UAVs as a sensor platform for SAR and GPR is not the only use for radar. Radar can provide reliable data where state-of-the-art sensors attached to UAVs reach their limits, e.g., in case of low contrast or back light in vision-based systems. It has been shown that measuring the altitude AGL is a simple task with a radar sensor. Additionally, detailed sections of a terrain profile can be obtained with a single radar measurement, using a $3 \times 8$ channel TDM MIMO radar with $2 \mathrm{GHz}$ bandwidth at a center frequency of $76.5 \mathrm{GHz}$ and chirp-sequence frequency modulation.

Further experiments with the imaging radar facing forward show the capability of surveying a broad area in front of the UAV with the ability of simultaneous multitarget range, velocity, and angle measurement. Targets in an angular range of up to $\pm 60^{\circ}$ in azimuth have been detected. Even small UAVs passing by could be observed. With appropriate tracking algorithms and trajectory estimation, collision avoidance and evasive maneuver could be realizable.

With the integration of complete radar frontends on single chips, which are becoming available in the ISM-bands at $60 \mathrm{GHz}$ and $122 \mathrm{GHz}$ and the results of the here presented survey, radar can increase sensing capability and safety of UAVs. The relative high frequencies enable small and light weight sensors, making radar feasible even for small UAVs.

\section{Acknowledgment}

This work was partly supported by the Ministry for Science, Research, and Arts BadenWürttemberg within the project ZAFH MikroSens. 


\section{References}

[1] S. Jordan, J. Moore, S. Hovet, J. Box, J. Perry, K. Kirsche, D. Lewis, and Z. T. H. Tse, "State-of-the-art technologies for UAV inspections," IET Radar, Sonar $\mathscr{E}$ Navigation, vol. 12, pp. 151-164, Feb. 2018.

[2] S. Krause, F. Hartmann, and J.-P. Mund, "UAV Workflow Optimization for the Acquisition of High-Quality Photogrammetric Point Clouds in Forestry," in GIForum: Journal for Geographic Information Science, vol. 1, 2016, pp. 72-84.

[3] J. Zhang, J. Hu, J. Lian, Z. Fan, X. Ouyang, and W. Ye, "Seeing the forest from drones: Testing the potential of lightweight drones as a tool for long-term forest monitoring," Biological Conservation, vol. 198, pp. 60-69, 2016.

[4] Deutsche Post DHL, "DHL parcelcopter launches initial operations for research purposes," Sep. 2014.

[5] — _ "Successful Trial Integration of DHL Parcelcopter into Logistics Chain," May 2016.

[6] P. Patel, "Agriculture drones are finally cleared for takeoff," IEEE Spectrum, vol. 53, no. 11, pp. 13-14, Nov. 2016.

[7] DJI, AGRAS MG-1S User Manual V1.0, Oct. 2017.

[8] M. Schartel, R. Burr, P. Schoeder, G. Rossi, P. Hügler, W. Mayer, and C. Waldschmidt, "Radar-Based Altitude over Ground Estimation of UAVs," in German Microwave Conference (GeMiC), Mar. 2018.

[9] M. Schartel, R. Burr, W. Mayer, N. Docci, and C. Waldschmidt, "UAV-Based Ground Penetrating Synthetic Aperture Radar," in IEEE MTT-S International Conference on Microwaves for Intelligent Mobility (ICMIM), 2018.

[10] DJI, MAVIC AIR User Manual V1.2, Feb. 2018.

[11] P. Hügler, M. Geiger, and C. Waldschmidt, " $77 \mathrm{GHz}$ Radar-Based Altimeter for Unmanned Aerial Vehicles," in IEEE Radio and Wireless Symposium (RWS), Jan. 2018, pp. 129-132.

[12] DJI, AGRAS MG-1S Obstacle Avoidance Radar V1.0, Jun. 2017.

[13] H. Essen, W. Johannes, S. Stanko, R. Sommer, A. Wahlen, and J. Wilcke, "HIGH RESOLUTION W-BAND UAV SAR," in IEEE International Geoscience and Remote Sensing Symposium, Jul. 2012, pp. 5033-5036.

[14] C. J. Li and H. Ling, "Synthetic Aperture Radar Imaging Using a Small Consumer Drone," in IEEE International Symposium on Antennas and Propagation USNC/URSI National Radio Science Meeting, Jul. 2015, pp. 685-686. 
[15] G. Fasano, A. Renga, A. R. Vetrella, G. Ludeno, I. Catapano, and F. Soldovieri, "Proof of concept of micro-UAV-based radar imaging," in International Conference on Unmanned Aircraft Systems (ICUAS), Jun. 2017, pp. 1316-1323.

[16] G. Ludeno, I. Catapano, G. Gennarelli, F. Soldovieri, A. R. Vetrella, A. Renga, and G. Fasano, "A micro-UAV-borne system for radar imaging: A feasibility study," in 9th International Workshop on Advanced Ground Penetrating Radar (IWAGPR), Jun. 2017, pp. 1-4.

[17] M. Schuetz, M. Oesterlein, C. Birkenhauer, and M. Vossiek, "A Custom Lightweight UAV for Radar Remote Sensing: Concept Design, Properties and Possible Applications," in IEEE MTT-S International Conference on Microwaves for Intelligent Mobility (ICMIM), Mar. 2017, pp. 107-110.

[18] A. Moses, M. J. Rutherford, M. Kontitsis, and K. P. Valavanis, "UAV-borne X-band radar for collision avoidance," Robotica, vol. 32, no. 1, p. 97-114, Jul. 2014.

[19] A. F. Scannapieco, A. Renga, G. Fasano, and A. Moccia, "Ultralight radar sensor for autonomous operations by micro-UAS," in International Conference on Unmanned Aircraft Systems (ICUAS), Jun. 2016, pp. 727-735.

[20] W. Liu, C. Yu, X. Wang, Y. Zhang, and Y. Yu, "The Altitude Hold Algorithm of UAV Based on Millimeter Wave Radar Sensors," in 9th International Conference on Intelligent Human-Machine Systems and Cybernetics (IHMSC), vol. 1, Aug. 2017, pp. 436-439.

[21] J. Hasch, E. Topak, R. Schnabel, T. Zwick, R. Weigel, and C. Waldschmidt, "Millimeter-Wave Technology for Automotive Radar Sensors in the $77 \mathrm{GHz}$ Frequency Band," IEEE Transactions on Microwave Theory and Techniques, vol. 60, no. 3, pp. 845-860, Mar. 2012.

[22] C. Waldschmidt and H. Meinel, "Future trends and directions in radar concerning the application for autonomous driving," in 44th European Microwave Conference, Oct. 2014, pp. 1719-1722.

[23] C. Beck, H. J. Ng, R. Agethen, M. PourMousavi, H. P. Forstner, M. Wojnowski, K. Pressel, R. Weigel, A. Hagelauer, and D. Kissinger, "Industrial mmWave Radar Sensor in Embedded Wafer-Level BGA Packaging Technology," IEEE Sensors Journal, vol. 16, no. 17, pp. 6566-6578, Sep. 2016.

[24] A. Hagelauer, M. Wojnowski, K. Pressel, R. Weigel, and D. Kissinger, "Integrated systems-in-package: Heterogeneous integration of millimeter-wave active circuits and passives in fan-out wafer-level packaging technologies," IEEE Microwave Magazine, vol. 19, no. 1, pp. 48-56, Jan. 2018.

[25] M. Hitzler, L. Boehm, W. Mayer, and C. Waldschmidt, "Radiation Pattern Optimization for QFN Packages with On-Chip Antennas at $160 \mathrm{GHz}$," IEEE Transactions on Antennas and Propagation, pp. 1-1, 2018. 
[26] V. Winkler, "Range Doppler detection for automotive FMCW Radars," in European Radar Conference (EuRAD), Oct. 2007, pp. 166-169.

[27] C. Schroeder and H. Rohling, "X-band FMCW Radar System with Variable Chirp Duration," in IEEE Radar Conference, May 2010, pp. 1255-1259.

[28] J. J. M. de Wit, W. L. van Rossum, and A. J. de Jong, "Orthogonal Waveforms for FMCW MIMO Radar," in IEEE RadarCon (RADAR), May 2011, pp. 686-691.

[29] R. Schmidt, "Multiple Emitter Location and Signal Parameter Estimation," IEEE Transactions on Antennas and Propagation, vol. 34, no. 3, pp. 276-280, Mar. 1986.

[30] P. Stoica and K. C. Sharman, "Maximum Likelihood Methods for Direction-ofArrival Estimation," IEEE Transactions on Acoustics, Speech, and Signal Processing, vol. 38, no. 7, pp. 1132-1143, Jul. 1990.

[31] P. Häcker and B. Yang, "Single snapshot DOA estimation," Advances in Radio Science, vol. 8, pp. 251-256, 2010.

[32] E. Topak, J. Hasch, and T. Zwick, "Compact Topside Millimeter-Wave Waveguideto-Microstrip Transitions," IEEE Microwave and Wireless Components Letters, vol. 23, no. 12, pp. 641-643, Dec. 2013.

[33] C. Vasanelli, R. Batra, A. D. Serio, F. Boegelsack, and C. Waldschmidt, "Assessment of a Millimeter-Wave Antenna System for MIMO Radar Applications," Antennas and Wireless Propagation Letters, vol. 16, pp. 1261-1264, 2017.

[34] C. Schmid, R. Feger, C. Pfeffer, and A. Stelzer, "Motion Compensation and Efficient Array Design for TDMA FMCW MIMO Radar Systems," in 6th European Conference on Antennas and Propagation (EUCAP), Mar. 2012, pp. 1746-1750.

[35] J. Bechter, F. Roos, and C. Waldschmidt, "Compensation of Motion-Induced Phase Errors in TDM MIMO Radars," IEEE Microwave and Wireless Components Letters, vol. 27, no. 12, pp. 1164-1166, Dec. 2017.

[36] F. Roos, J. Bechter, N. Appenrodt, J. Dickmann, and C. Waldschmidt, "Enhancement of Doppler Unambiguity for Chirp-Sequence Modulated TDM-MIMO Radars," in International Conference on Microwaves for Intelligent Mobility (ICMIM), Apr. 2018. 